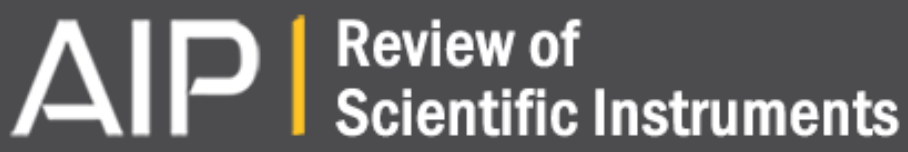

\section{Detection of high $\mathrm{k}$ turbulence using two dimensional phase contrast imaging on}

\section{LHDa)}

C. A. Michael, K. Tanaka, L. N. Vyacheslavov, A. Sanin, N. K. Kharchev, T. Akiyama, K. Kawahata, and S. Okajima

Citation: Review of Scientific Instruments 79, 10E724 (2008); doi: 10.1063/1.2968266

View online: http://dx.doi.org/10.1063/1.2968266

View Table of Contents: http://scitation.aip.org/content/aip/journal/rsi/79/10?ver=pdfcov

Published by the AIP Publishing

\section{Articles you may be interested in}

Short-interval multi-laser Thomson scattering measurements of hydrogen pellet ablation in LHDa)

Rev. Sci. Instrum. 85, 11D822 (2014); 10.1063/1.4890251

Perpendicular and tangential angularly resolved multi-sight neutral particle analyzer system in LHDa)

Rev. Sci. Instrum. 83, 10D920 (2012); 10.1063/1.4742925

Microwave Doppler reflectometer system in LHDa)

Rev. Sci. Instrum. 83, 10E322 (2012); 10.1063/1.4733736

Spectroscopic diagnostics for ablation cloud of tracer-encapsulated solid pellet in LHDa)

Rev. Sci. Instrum. 79, 10F541 (2008); 10.1063/1.2957928

An analysis of the influence of impurities on fast particle attenuation and on fast ion spectral shape in LHDa)

Rev. Sci. Instrum. 79, 10F310 (2008); 10.1063/1.2953673

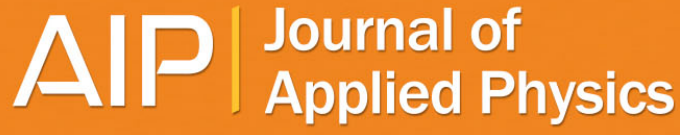

Journal of Applied Physics is pleased to announce André Anders as its new Editor-in-Chief 


\title{
Detection of high $k$ turbulence using two dimensional phase contrast imaging on LHD ${ }^{a)}$
}

\author{
C. A. Michael, ${ }^{1}$ K. Tanaka, ${ }_{1}^{1}$ L. N. Vyacheslavov,${ }^{2}$ A. Sanin, ${ }^{2}$ N. K. Kharchev, ${ }^{3}$ \\ T. Akiyama, ${ }^{1}$ K. Kawahata, ${ }^{1}$ and S. Okajima ${ }^{4}$ \\ ${ }^{1}$ National Institute for Fusion Science, 322-6 Oroshi-cho, Toki 509-5292, Japan \\ ${ }^{2}$ Budker Institute of Nuclear Physics, 630090 Novosibirsk, Russia \\ ${ }^{3}$ A.M. Prokhorov General Physics Institute, Moscow 119991, Russia \\ ${ }^{4}$ Department of Engineering, Chubu University, Kasugai 487-8501, Japan
}

(Presented 13 May 2008; received 12 May 2008; accepted 17 July 2008;

published online 31 October 2008)

\begin{abstract}
High $k$ turbulence, up to $30 \mathrm{~cm}^{-1}$, can be measured using the two dimensional $\mathrm{CO} 2$ laser phase contrast imaging system on LHD. Recent hardware improvements and experimental results are presented. Precise control over the lens positions in the detection system is necessary because of the short depth of focus for high $k$ modes. Remote controllable motors to move optical elements were installed, which, combined with measurements of the response to ultrasound injection, allowed experimental verification and shot-to-shot adjustment of the object plane. Strong high $k$ signals are observed within the first 100-200 ms after the initial electron cyclotron heating (ECH) breakdown, in agreement with gyrotron scattering. During later times in the discharge, the entire $k$ spectrum shifts to lower values (although the total amplitude does not change significantly), and the weaker high $k$ signals are obscured by leakage of low $k$ components at low frequency, and detector noise, at high frequency. () 2008 American Institute of Physics. [DOI: 10.1063/1.2968266]
\end{abstract}

\section{INTRODUCTION}

Transport in high temperature plasmas is often dominated by "anomalous" processes, thought to be due to the presence of turbulence at the ion and electron gyroscale. Although much attention has been given to ion gyroscale instabilities, recent interest has been directed toward smaller scale instabilities, in particular, the so-called electrontemperature gradient mode. The challenge from a diagnostic point of view is to improve the sensitivity to smaller amplitude, smaller wavelength structures. Diagnostics developed for measuring high $k$ turbulence include microwave scattering ${ }^{1-3}$ and phase contrast imaging (PCI). ${ }^{4,5}$ On LHD, a high $k$ scattering system employing a heating gyrotron as a source is installed ${ }^{6}$ and is compared to PCI results presented here.

A two dimensional (2D) CO2 laser PCI system was installed on the Large Helical Device (LHD) ${ }^{7,8}$ This uses the novel principle of $2 \mathrm{D}$ imaging, taking advantage of the strong angular shear of field lines from different points within the plasma to resolve the spatial dependence of fluctuation properties. Specifically, by measuring the 2D propagation vector $\left(k_{x}, k_{y}\right)$, the angle $\tan ^{-1}\left(k_{y} / k_{x}\right)$ $=\tan ^{-1}\left[B_{y}(z) / B_{x}(z)\right]+\pi / 2$ is related uniquely to $z$, since fluctuations propagate perpendicular to field lines (where axes $x$ and $y$ are aligned along the inverse image of the detector array in the plasma and $z$ is a coordinate along the beam). It was upgraded two years $\mathrm{ago}^{4}$ to use a wider beam to detect lower $k$ and gain better spatial resolution and equipped with a flexible imaging system, featuring a Dove image rotator,

\footnotetext{
a) Contributed paper, published as part of the Proceedings of the 17th Topical Conference on High-Temperature Plasma Diagnostics, Albuquerque, New Mexico, May 2008.
}

translatable roof mirror, and interchangeable cylindrical/ spherical lenses to change the aspect ratio and demagnification. This enables the Nyquist $k$ in the $x$ and $y$ directions $k_{x}^{\text {nyq }}, k_{y}^{\text {nyq }}$ to be varied. When the Nyquist $k$ boundary is converted from Cartesian to polar coordinates, $k=\sqrt{k_{x}^{2}+k_{y}^{2}}, \theta$ $=\tan ^{-1}\left(k_{y} / k_{x}\right), k^{\text {nyq }}$ increases from $k_{x}^{\text {nyq }}$ at $\theta=0$ to a maximum value of $\sqrt{\left(k_{x}^{\text {nyq }}\right)^{2}+\left(k_{y}^{\text {nyq }}\right)^{2}}$ at $\theta=\theta_{c}=\tan ^{-1}\left(k_{y}^{\text {nyq }} / k_{x}^{\text {nyq }}\right)$, then decreases to $k_{y}^{\text {nyq }}$ at $\theta=\pi / 2$. Because $k_{y}^{\text {nyq }}$ is chosen less than $k_{x}^{\text {nyq }}$, there is a particular angular range from $-\theta_{c}<\theta<\theta_{c}$, where the Nyquist $k$ is higher and the spatial resolution is better. ${ }^{4}$ The angle $2 \theta_{c}$ characterizes the measurable angular range with good resolution.

The aspect ratio of the rectangular sampled region in the plasma can be varied by reconfiguring the imaging system. According to the original design, ${ }^{4}$ the imaging system could be used in "overview mode," employing spherical lenses to achieve a uniform $x$ and $y$ demagnification of $m_{x}=m_{y}=7.8$ to detect fluctuations along the entire line of sight, with $k^{\text {min }}$ $=1 \mathrm{~cm}^{-1}, k_{x}^{\text {nyq }}=6 \mathrm{~cm}^{-1}$, and $k_{y}^{\text {nyq }}=3 \mathrm{~cm}^{-1}$, so that the $2 \theta_{c}$ $=53^{\circ}$ approximately matched to $\theta\left(z_{\text {top }}\right)-\theta\left(z_{\text {bottom }}\right)=80^{\circ}$. Alternatively, the system could be adjusted to "zoom mode," employing a pair of cylindrical lenses to demagnify the object plane by a factor along the field lines of $m_{y}=7.8$ and across the field lines only by a factor $m_{x}=1.6$, giving $k_{x}^{\text {nyq }}$ $=30 \mathrm{~cm}^{-1}$ and $k_{y}^{\text {nyq }}=3 \mathrm{~cm}^{-1}$, for sensing high $k$ fluctuations only within only a small angular region of $2 \theta_{c}=11^{\circ}$, corresponding to a length along the line of sight $2 \theta_{c} d z / d \theta$ of around $25 \mathrm{~cm}$ (where the total interaction length through the plasma is around $2 \mathrm{~m}$ ). The particular part of the plasma targeted can be tuned by turning the dove image rotator. Concomitantly, it is necessary to move object plane to the place where the angle of the Dove rotator matches the angle 
of the field lines, in particular, for high $k$ fluctuations since the depth of focus is considerably smaller than the length through the plasma. This is adjusted by moving a roof mirror installed between the phase plate and intermediate image to change the optical path length. (Slight modifications were made to the magnification of overview mode as well as zoom mode, described in Sec. III.)

A description of the system, analysis techniques, and summary of results is described in these proceedings. ${ }^{9}$ Though high $k$ results were initially presented, ${ }^{4}$ the high $k$ components were obscured by low $k$ ones, necessitating a very high frequency filter $f>1.2 \mathrm{MHz}$ to isolate high $k$. At this time attention was not given to the tight tolerances required, and little discussion was given to the issues about detection of high $k$. The purpose of this paper is to consider these issues (Sec. II), describe a few small improvements to the system (Sec. III), and show some new results (Sec. IV).

\section{DETECTABILITY OF HIGH $\boldsymbol{k}$}

We consider the "detectability" of high $k$ components to be the ratio of the high $k$ sensitivity to the obstructions from other components, including systematic errors: leakage from low $k$ to high $k$, detector noise, and laser noise.

The main issue affecting the sensitivity of high $k$ components is blurring of the fluctuation image, which is quantified by the ratio of the distance between the fluctuation and the object plane (of the imaging system) and the depth of focus. However, the depth of focus has a different effect for pointlike structures as it does for wavelike structures. For pointlike structures, the image is simply blurred and there is a continuous loss of contrast with increasing distance out of focus. For detecting wavelike structures with PCI, however, constructive or destructive interference (scintillation effects) can occur which makes the contrast $s$ of the object (in the center of the beam) vary periodically with distance $L$ between the object plane and the fluctuation, according to the Talbot effect: ${ }^{10-12} s=\left|\cos \left(\pi L / 2 L_{T / 4}\right)\right|$, where $L_{T / 4}=2 \pi^{2} / \lambda_{0} k^{2}$ is the quarter Talbot length for a given value fluctuation wavenumber $k$ and collimated probing beam wavelength $\lambda_{0}$. This is the distance at which a fluctuation must be out of focus in order to produce maximum cancellation or minimum contrast. For $k=30 \mathrm{~cm}^{-1}, L_{T / 4}=20 \mathrm{~cm}$. Since this is smaller than the diameter of the plasma $(\sim 2 \mathrm{~m})$ high $k$ fluctuations cannot be simultaneously detected from all positions along the beam. The position of the object plane is determined by the distances between lenses in the imaging system. A translation of the roof mirror by $\Delta$ (or equivalently an error in the position of other optical elements by $\Delta / 2$ ) causes the object plane to move by $32 \Delta$. Therefore, to be correct within the Talbot length of $20 \mathrm{~cm}, \Delta$ must be set accurately to within $7 \mathrm{~mm}$. Combined placement errors of a few millimeter on the optical bench can constitute such a displacement. Therefore it is essential to carefully position all the imaging optics and check it with ultrasound measurements.

The leakage of low $k$ components into high $k$ is the most serious obstruction to high $k$ components; for this reason the signal to noise ratio (SNR) is not the most important factor (so long as it exceeds a certain value). Large and small scale fluctuations need to be separated in software using the spatial Fourier transform. Although in analysis, the sensitivity of each channel is normalized such that the autopower spectrum is the same for all channels, the cross-correlation function between pairs of channels with the same fixed separation can show several percent variation. This characteristic "error" may cause leakage from low $k$ to high $k$ of order a few percent. In fact, the leakage may even arise from a fluctuation at a different position. The physical origin of this inconsistency may be due to scattering or diffraction off optical elements which will effectively produce a finite power density over a patch of the phase plate, which produces a broadband $k$ leakage.

This leakage and noise has the following relationship to the maximum detectable $k$ (for that frequency interval). Firstly, according to the line integration effect, $\widetilde{N}^{2}=\widetilde{n}^{2} l_{z} L_{R}$, where $\tilde{N}$ is the line integrated fluctuation amplitude, $\tilde{n}$ is the local fluctuation amplitude, $l_{z} \propto 1 / k$ is related to the correlation length and/or wavelength, and $L_{R}$ is the resolution, however, $L_{R} \propto 1 / k$ because higher $k$ components have better angular separation. Therefore, $\tilde{N}^{2} \propto \tilde{n}^{2} k^{-2}$. Because of the unfolding of the line integration effect, a flat broadband $k$ raw signal noise component (which may have contributions from leakage from strong values of $k$ as well as genuine noise) $\widetilde{N}_{\text {noise }}^{2} \propto k^{0}$ implies $\widetilde{n}_{\text {noise }}^{2} \propto k^{2}$, increasing with $k$. Supposing that the local fluctuation amplitude follows a power law $\tilde{n}^{2} \propto k^{-\alpha}$, then $\tilde{N}^{2} \propto k^{-\alpha-2}$. Defining $R=\tilde{N}^{2}\left(k_{0}\right) / \tilde{N}_{\text {noise }}^{2}$, where $k_{0}$ is the peak $k$, then the maximum $k$ where the $\mathrm{SNR} \geqslant 1$ is $k_{\max }=k_{0} R^{1 /(\alpha+2)}$. The power law dependence and noise floor are clear in experimental data in Sec. IV. Using a temporal frequency filter is one obvious method to reject lower $k$ components, however, depending on the nature of the dispersion relation, this may or may not correctly isolate higher $k$ components. For example, as the power decreases with frequency, the signal starts to be comparable with the uncorrelated detector noise. Laser noise can be filtered out digitally as it is coherent on all channels, having the structure of $k=0$ component.

\section{RECENT IMPROVEMENTS TO SYSTEM}

(1) Adjustment of magnification and aspect ratio to control the measurable angular range $\left(2 \theta_{c}\right)$ and power density for optimum signal to detector noise ratio. Since the width of the probing laser beam is set to be as large as possible, compatible with the capability to measure low $k$, the power density of the probing beam cannot be increased without an entire realignment of the beam. When switching the detection optics from overview mode to high $k$ zoom mode, changes in the demagnification can have a profound effect on the power density at the detector, and hence the SNR. The most appropriate power density for the detector is around $4 \mathrm{~mW} / \mathrm{mm}^{2}$. For $m_{x}=m_{y}=7.8$ (overview mode), the power density is so high that the beam needs attenuation using a 17\%/83\% beam splitter. On the other hand, if spherical lenses alone were used to set $m_{x}=m_{y}=1.56$ giving $k_{x}^{\mathrm{nyq}}=30 \mathrm{~cm}^{-1}$ and $k_{y}^{\mathrm{nyq}}=15 \mathrm{~cm}^{-1}$, giving a measurable angular range of $2 \theta_{c}=53^{\circ}$, then the power den- 
sity is too low on the detector (around $1 \mathrm{~mW} / \mathrm{mm}^{2}$ ). Such an option might be attractive because the small angular range of zoom mode often proves to be too restrictive. Finally, a demagnification of $m_{x}=1.56, m_{y}$ $=3.9$ was decided, giving a factor of 2.5 higher power density on the detector (than $m_{x}=m_{y}=1.56$ ) and reduces the angular range to around $2 \theta_{c}=23^{\circ}$, which translates to a range along the beam, $2 \theta_{c} d z / d \theta=58 \mathrm{~cm}$, which is about three times the quarter Talbot length at $k$ $=30 \mathrm{~cm}^{-1}$. This magnification gives near the optimum power density. The magnification of this configuration is slightly different from the originally designed zoom mode. $^{4}$

(2) A motor drive was attached to the image rotator and roof mirror system to enable the object plane and targeted field line angle to be varied between shots. This was helpful since it is possible to intervene manually only at the end of every day, and similar shots are generally not obtained on successive days because of change in the physics plan. With the motor drive system, the targeted position in the plasma can be varied between shots. However, the Dove rotator/roof mirror system must be aligned very accurately such that the beam walk off on the phase plate and on the detector, as the rotator/roof mirror is moved within a tolerable limit. Under manual intervention, any beam walk-off could be compensated for by simply moving the phase plate and/or detector array. The most stringent walk-off tolerance is the phase plate, since the width of the groove is set around $0.5 \mathrm{~mm}$. An iterative alignment procedure was performed, adjusting the $x / y$ steering controls of a pair of relay mirrors.

(3) A spatial filter mask in the Fourier plane is not used. This was used in Ref. 4 to reject signals from outside the measured $k$ band that would be subject to aliasing, thereby giving the incorrect location and/or value of $k$. However, since the measurable angular range $2 \theta_{c}$ is narrow in zoom mode, it is informative to collect the signal from components propagating at an angle slightly outside the domain $\left[\theta_{0} \theta_{c}, \theta_{0}+\theta_{c}\right]$ where $\theta_{0}$ is angle of dove rotor. Because there are often only several clearly distinguishable components (in terms of spatial location, propagation direction) and they conform approximately with a linear $k$ /frequency relationship [see Fig. 3], the aliased components can most of the time be identified clearly because they break the trend (in particular, that $|k|$ decreases with frequency). Moreover, since aliasing is only possible in the $y$ direction (since $k_{y}^{\text {nyq }} \ll k_{x}^{\text {nyq }}$ ), $|k|$ of the aliased component is less than or equal to $|k|$ of the unaliased one. Hence, the highest $k$ components do not suffer contamination. In fact, the more intense low $k$ components cause more serious contamination of high $k$ components through leakage. A spatial filter mask which rejects these low $k$ components may be important in the future but was not used here.

(4) A variable frequency ultrasound speaker was purchased for investigating the Talbot effect and confirming the object position location; previously fixed frequency resonant devices were used. However, since the location of the sound injection cannot be changed easily, it is much more useful to have a variable frequency source.

\section{CONFIRMATION OF OBJECT PLANE POSITION WITH ULTRASONIC SPEAKER}

An independent measurement using ultrasound of the position of the object plane gives good confidence in the capability to measure high $k$. The objective here is to experimentally determine the distance $L$ between the object plane and the known position of an ultrasound wave (located $6.87 \mathrm{~m}$ from the center of the plasma) and measure the displacement of the roof mirror which locates the object plane at the center of the plasma. By varying the ultrasonic frequency, the wavenumber can be scanned, and so according to the Talbot effect as discussed in Sec. II, the contrast (ac signal intensity) for a collimated beam should vary as $s=\left|\cos \left(\pi L \lambda_{0} f^{2} / v^{2}\right)\right|$, where $v$ is the speed of sound. Therefore, the frequencies where $s$ has a local minimum can be used to determine $L$. Because the frequency interval between successive minima is less at higher frequency, higher frequencies give better accuracy in $L$ (although more degeneracy). Because the injected beam is not collimated presently, a wave-optics beam propagation code (GLAD) was used to obtain a more accurate result. In the model for the detection optics, a displacement $\Delta$ of the roof mirror is introduced as a parameter to represent the effect of shifting the object plane away from the center of the plasma. The value $\Delta=0$ represents the designed position of the roof mirror such that the object plane is in the center of the plasma. As the contrast depends on the position within the beam cross section, the representative "intensity" was taken as the integral over the entire beam profile. A contour plot of the calculated intensity response as a function of frequency and displacement $\Delta$ is plotted in Fig. 1(b). This is to be compared to the measured PCI frequency response to the injected sound wave (appropriately filtered), summed over all channels, as plotted in Fig. 1(a). The experimental value of the frequency of the Talbot minimum in the range $f=100-110 \mathrm{kHz}$ is $f_{\text {min }}$ $=105 \mathrm{kHz}$. According to the calculation result, $f_{\min }$ $=105 \mathrm{kHz}$ for $\Delta=0.42 \mathrm{~cm}$. (Other possible values include $+2.1 \mathrm{~cm}$ and $-1.3 \mathrm{~cm}$, however, these are inconsistent with other measurements at lower frequency.) Thus the object plane is away from the center of the plasma by approximately $13.5 \mathrm{~cm}$, more than half of the quarter Talbot length (at $k=30 \mathrm{~cm}^{-1}$ ). This value was subsequently used to correct the roof mirror displacement so that the object plane could be more accurately positioned.

\section{MEASURED HIGH $\boldsymbol{k}$ SIGNALS}

High $k$ turbulence was detected during the ECH breakdown phase, within about 100-200 ms of the start of the $\mathrm{ECH}$ pulse [before neutral beam injection (NBI)]. The presence of high $k$ during the same ECH phase was also confirmed with gyrotron scattering. ${ }^{6}$ In order to confirm the Talbot effect on the sensitivity to high $k$, the Dove rotator was oriented along the field lines in the center, giving a measurable radial range from -0.4 to +0.4 , and the position of the 
(a) measured intensity response

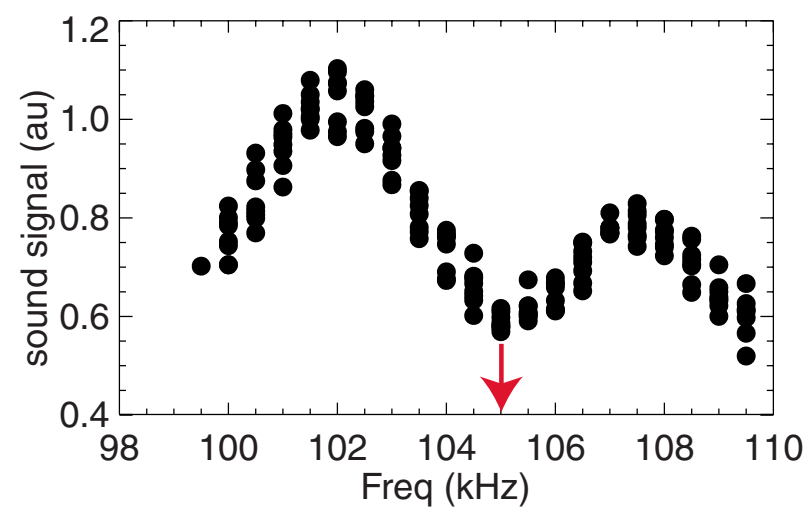

(b) Calculated intensity response

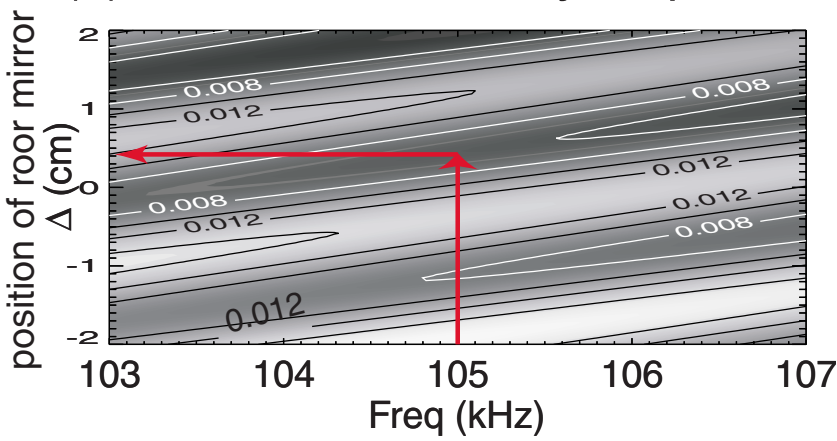

FIG. 1. (Color online) (a) Measured response to ultrasound frequency sweep; summed over all channels, to compare to (b) calculated intensity response, integrated over the entire beam, as a function of sound frequency for and the error in the roof mirror position $\Delta$. The experimental minimum at $f=105 \mathrm{kHz}$ implies that $\Delta=0.42 \mathrm{~cm}$.

object plane was moved from $\rho=+0.3$ (above the midplane) to $\rho=-0.3$ (below the midplane). The spatial $/ k$ variation of fluctuation amplitude for each case is plotted in Fig. 2. The thicker dashed line indicates the object plane in each figure, and the red line indicates positions which are exactly a quarter Talbot length away from the object plane (which is itself

(a)

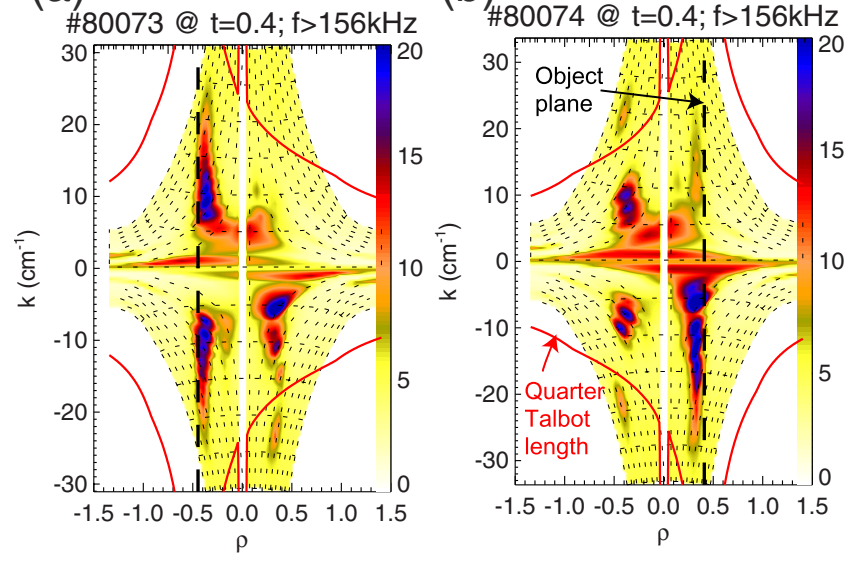

FIG. 2. (Color online) Images of the spatial and $k$ dependence of fluctuation amplitude for $f>156 \mathrm{kHz}$ during the ECH breakdown phase, for successive shots where the object plane is moved from (a) $\rho=-0.4$ and (b) $\rho=+0.4$. In each figure, thick dashed lines denote the position of the object plane, and the red line denotes the the quarter Talbot distance. The dashed grid characterizes the spatial $/ k$ resolution by sampling considerations.

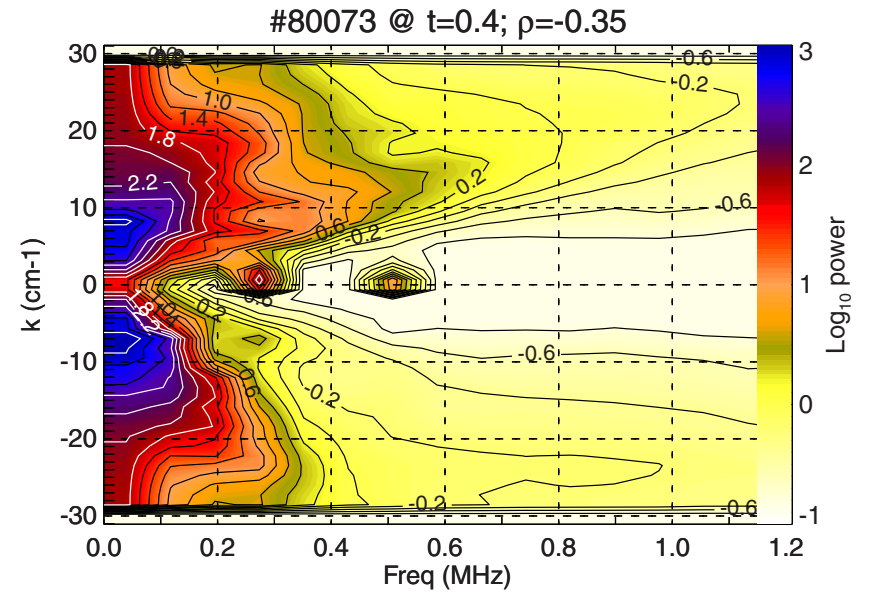

FIG. 3. (Color online) Dispersion relation for components at $\rho=-0.35$, for 80073 , where the object plane was set to $\rho=-0.4$, and the plasma was in the $\mathrm{ECH}$ breakdown phase.

dependent on $k$ ). It can be seen that turbulence propagating in both directions is observed at $\rho=0.3$ both above and below the midplane, and that the signal is strongest at the location of the object plane (further confirmation that the object plane is correct). Additionally, a distinct dip in the $k$ spectrum is evident for $k \sim 20 \mathrm{~cm}^{-1}$ in Fig. 2(a), at $\rho=0.3$, corresponding to the $k$ for which the distance to the object plane is equal to the quarter Talbot length. The characteristics of the high $k$ turbulence are shown more clearly by examining the dispersion relation at the object plane in Fig. 3. Power extends up to the Nyquist $k$ clearly around $300-400 \mathrm{kHz}$, while at below $100 \mathrm{kHz}$ the amplitude does not decrease with $k$ for $|k|$ $>20 \mathrm{~cm}^{-1}$, indicating possible limitation of the image dynamic range. This particular characteristic of the dispersion relation has also been observed in other PCI systems. ${ }^{13}$

While the presence of high $k$ during the ECH breakdown phase confirms the operation of the diagnostic, it is not of so much physics relevance, since during this phase, the plasma is forming, so steep temperature gradients (driving turbulence) are formed near the EC resonance location. (This speculation cannot be confirmed due to poor SNR of temperature measurements during the breakdown phase.) After ion-electron thermal equilibration and the thermal confinement time, the temperature gradient may become weaker reducing the turbulence drive. However, during the flat top of the discharge, the high $k$ turbulence is generally weaker and is obscured by leakage of low $k$ signals into higher $k$. When the object position and Dove rotator are scanned (on a shotto-shot basis) from the core to the edge, the only reliable signals are found where the turbulence is strongest, precisely around $\rho=-1$. A spatial $/ k$ map of the turbulence amplitude with the Dove rotator fixed so that the radial region is centered about $\rho=-1$ is plotted in Fig. 4. While the angular range $2 \theta_{c}$ is the same as for Fig. 2, the strong change in $d \theta / d \rho$ means that the radial range covered at the edge is much smaller. The direction of propagation reverses about $\rho=-1$. Furthermore, the amplitude at both $\rho=-0.95$ and 1.05 seems to increase with $k$, for $k>15 \mathrm{~cm}^{-1}$. Such an increase seems unlikely and may be due to an artificial broadband $k$ 


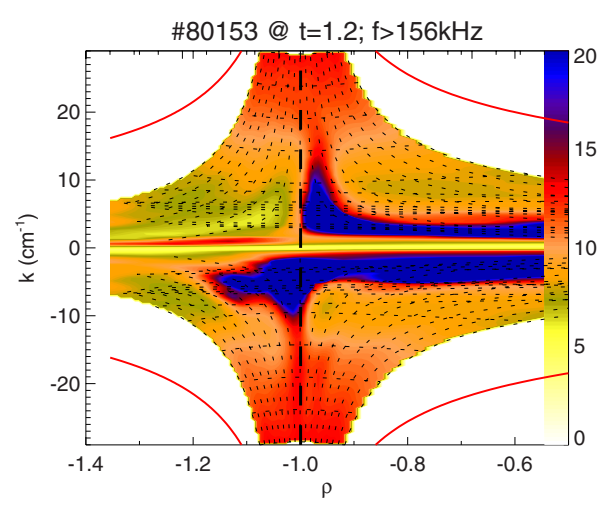

FIG. 4. (Color online) Image of the spatial and $k$ dependence of fluctuation amplitude for $f>156 \mathrm{kHz}$ during the flat-top phase of a discharge, where the object position and Dove rotator position were both set to be $\rho=-1$.

component, arising from the low $k$ leakage, which originates from the lower end of the frequency band here as discussed in Sec. II.

The $k$ spectra, plotted on a log-log scale, are compared between the ECH breakdown and NBI flat-top phases, with frequency filters $f>39 \mathrm{kHz}, f>312 \mathrm{kHz}$, and $f>625 \mathrm{kHz}$ applied in Figs. 5(a)-5(c), respectively. The noise level obtained from a period with no plasma is also indicated. In Fig. 5(a), the dynamic range $R$ as described in Sec. II is around 15 for both phases. The peak $k$ is around $4 \mathrm{~cm}^{-1}$ in the NBI phase and $6.3 \mathrm{~cm}^{-1}$ during the ECH breakdown phase. It also appears that the power rolls off to a noise floor defining

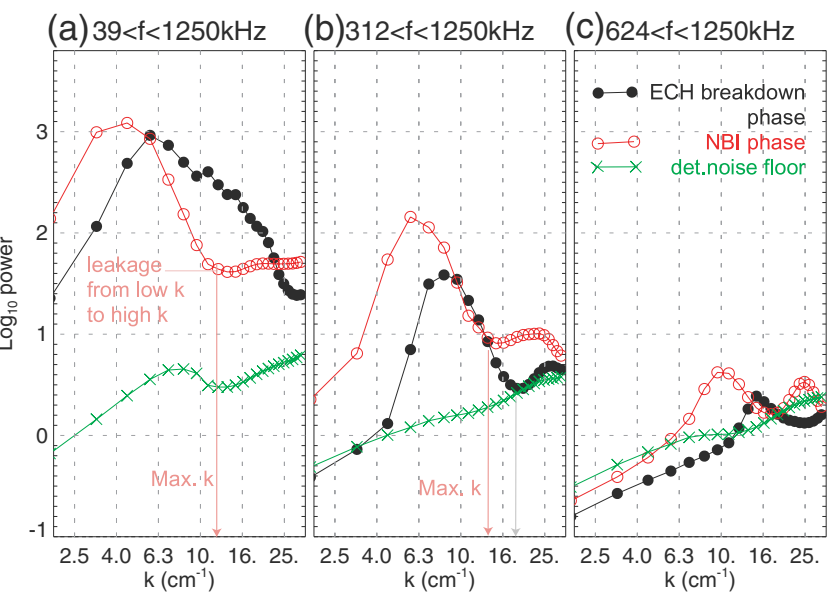

FIG. 5. (Color online) Comparsion of the $k$ spectra for different frequency intervals, between the ECH breakdown phase (80297 at $t=0.4 s ; \rho=-0.35$ ), the flat-top phase of the discharge (80153 at $t=1.2 s ; \rho=-1.05$ ), and the signal without plasma, attributable to detector noise (80297 at $t=0.15 \mathrm{~s}$; $\rho=-0.35$ ). $k_{\max }=15 \mathrm{~cm}^{-1}$ during the NBI phase, while there is no clear noise floor in the ECH breakdown phase. In Fig. 5(a), the power level at high $k$ is about 13 times larger than the noise level and so is likely to be due to leakage from low $k$ to higher $k$. For $f>312 \mathrm{kHz}$ in Fig. 5(b), the minimum signal level is only a few times larger than the noise level, while going to $f>624 \mathrm{kHz}$ in Fig. 5(c), most of the signals in both phases are dominated by noise except for a few peaks in the NBI phase (red) at around $k=28$ and $10 \mathrm{~cm}^{-1}$. However, as the amount of power at high frequency depends on the phase velocity of modes which is strongly affected by poloidal plasma rotation, there is no clearly defined frequency threshold for comparing different discharge types.

In Fig. 5(a), the larger $k_{\max }$ can be attributed to the larger peak $k$ and a slightly shallower slope (weaker exponent $\alpha$ ). One possible explanation for the shift in $k_{0}$ is that during the ECH breakdown phase, the ion temperature should be small, thereby having little drive for ion temperature gradient modes, which are located at lower $k$.

In the future, the use of a mask in the Fourier plane to cut the lower $k$ components to reduce leakage from low $k$ to high $k$ and may help improve detectability of high $k$ components. Reduction of small scale ripples in the beam profile, introduced by imperfections in the optics, could also help reduce the low $k$ leakage. Leakage can also arise in the Fourier analysis of the limited number of channels. It is clear that these issues are paramount to extending the range of measuring high $k$ in regular discharges. Strategies to improve the margin against laser noise such as the use of an attenuating phase plate may only help to detect high frequency components.

${ }^{1}$ E. Z. Gusakov et al., Plasma Phys. Controlled Fusion 48, B443 (2006).

${ }^{2}$ D. Smith et al., Rev. Sci. Instrum. 75, 3840 (2004).

${ }^{3}$ G. Batanov et al., Plasma Phys. Rep. 29, 363 (2003).

${ }^{4}$ C. Michael, K. Tanaka, L. Vyacheslavov, A. Sanin, K. Kawahata, and S. Okajima, Rev. Sci. Instrum. 77, 10E923 (2006).

${ }^{5}$ L. Lin et al., Rev. Sci. Instrum. 77, 10E918 (2006).

${ }^{6}$ N. Kharchev et al., Rev. Sci. Instrum. 79, 10E721 (2008).

${ }^{7}$ A. Sanin, K. Tanaka, L. Vyacheslavov, K. Kawahata, and T. Akiyama, Rev. Sci. Instrum. 75, 3439 (2004).

${ }^{8}$ L. Vyacheslavov, K. Tanaka, A. Sanin, K. Kawahata, C. Michael, and T. Akiyama, IEEE Trans. Plasma Sci. 33, 464 (2005).

${ }^{9}$ K. Tanaka et al., Rev. Sci. Instrum. 79, 10E702 (2008).

${ }^{10}$ H. Talbot et al., Philos. Mag. 9, 402 (1836).

${ }^{11}$ H. Wiesen, Plasma Phys. Controlled Fusion 28, 1147 (1986).

${ }^{12}$ K. Matsuo, K. Tanaka et al., Jpn. J. Appl. Phys., Part 1 30, 1102 (1991).

${ }^{13}$ M. Porkolab, J. C. Rost, N. Basse, J. Dorris, E. Edlund, L. Liang, Y. Lin, S. Wukitch et al., IEEE Trans. Plasma Sci. 34, 229 (2006). 